\title{
Modeling and dynamic analysis of novel coronavirus pneumonia (COVID-19) in China
}

\author{
Youming Guo ${ }^{1} \cdot$ Tingting $\mathrm{Li}^{1}$ \\ Received: 14 April 2021 / Revised: 17 July 2021 / Accepted: 19 July 2021 / \\ Published online: 23 September 2021 \\ (c) Korean Society for Informatics and Computational Applied Mathematics 2021
}

\begin{abstract}
Although novel coronavirus pneumonia (COVID-19) was widely spread in mainland China in early 2020, it was soon controlled. To study the impact of government interventions on the spread of disease during epidemics, a differential equation system is established to simulate the process of virus propagation in this paper. We first analyze its basic properties, basic reproduction number $R_{0}$ and existence of equilibria. Then we prove that the disease-free equilibrium (DFE) is Globally Asymptotically Stable when $R_{0}$ is less than 1 . Through the analysis of the daily epidemic data from January 10, 2020 to March 11, 2020, combined with the implementation of the national epidemic policy, we divide the whole process into three stages: the first stage (natural state), the second stage (isolation state), the third stage (isolation, detection and treatment). By using the weighted nonlinear least square method to fit the data of three stages, the parameters are obtained, and three basic reproduction numbers are calculated, which are: $R_{01}=2.6735, R_{02}=0.85077, R_{03}=0.18249$. Sensitivity analysis of threshold parameters and corresponding graphical results were also performed to examine the relative importance of various model parameters to the spread and prevalence of COVID-19. Finally, we simulate the trend of three stages and verify the theory of Global Asymptotic Stability of DFE. The conclusion of this paper proves theoretically that the Chinese government's epidemic prevention measures are effective in the fight against the spread of COVID-19. This study can not only provide a reference for research methods to simulate COVID-19 transmission in other countries or regions, but also provide recommendations on COVID-19 prevention measures for them.
\end{abstract}

This work was supported by the Basic Competence Promotion Project for Young and Middle-aged Teachers in Guangxi(2019KY0269).

$凶 \quad$ Tingting Li

wsltt0621@163.com

1 College of Science, Guilin University of Technology, Guilin 541004, Guangxi, People's Republic of China 
Keywords COVID-19 model $\cdot$ Stability $\cdot$ Parameter estimation · Basic reproduction number

Mathematics Subject Classification 34D23 • 49J15

\section{Introduction}

In 2020, the all world is facing a battle against a new type of viral pneumonia. The novel coronavirus pneumonia was named "COVID-19" (Corona Virus Disease 2019) by the director general of World Health Organization (WHO) Tedros on February 11, 2020 [1,2]. Meanwhile, International Committee on Taxonomy of Viruses declared that the novel coronavirus was named "SARS-CoV-2" (Severe Acute Respiratory Syndrome Coronavirus 2), and it was identified as a sister virus of SARS coronavirus. The main transmission route of COVID-19 is respiratory droplets transmission and contact transmission. It is very infectious, but the death rate is not high. As the outbreak continues to worsen, on 28 February 2020, the WHO raised the regional and global risk levels to the highest level, "very high", in its daily COVID-19 situation report. As of 11 March 2020, COVID-19 has spread to 115 countries, with 119,239 confirmed cases and 4,287 deaths. On the same day, the director general of the WHO, Tedros, announced that based on the assessment, WHO believes that the current outbreak of COVID-19 can be called a global pandemic [3].

In early January 2020, a new cases of viral pneumonia were reported in Wuhan [4]. With a large number of people moving during the Spring Festival, the number of infected cases increased rapidly. After finding the evidence of human-to-human transmission, the Chinese government issued a blockade of Wuhan on January 23, 2020 , in order to control the number of infected people. All the citizens actively cooperated with home quarantine, which greatly reduced the flow of people and the spread of the virus. Although a prolonged urban blockade would cause great damage to the economy, the Chinese government immediately implemented the measures of "trapping and killing the virus" in a responsible attitude towards the people and the world. By mid-march, the number of new confirmed cases nationwide had dropped to single digits. The spread of the virus in China has been basically contained.

Many domestic and foreign epidemiologists have done a lot of modeling and prediction on the spread of COVID-19 in China during the outbreak [5-12,29]. Such as: Using data from reported confirmed cases before 22 January 2020, Tang et al. [5] estimated that the basic reproduction number could be as high as 6.47 based on likelihood analysis and model analysis. Yan et al. [6] proposed a mathematical model of COVID-19 with time delay based on the cumulative confirmed number and cured cases published by the National Health Commission. The time delay is introduced to describe the incubation period of the virus. Khan and Atangana [7] established a fractional mathematical model to simulate the spread of COVID-19, estimated the parameters through the confirmed cases of 21 January to 28 January, and analyzed the sensitivity of the basic reproduction number. Due to the implementation of a series of strong intervention policies issued by the state, Tang et al. [8] used the new data as of 
January 29 to re-estimate the parameters of the model, and calculated that the basic reproduction number was less than 1 .

With the continuous spread of COVID-19 around the world, many scholars established a series of COVID-19 models to simulate its spread according to different situations and considering different influencing factors [39-47]. Aba et al. [39] developed a fractional mathematical model for the dynamics of COVID-19 by taking into account quarantine, isolation, and environmental viral load factors. Then, by fitting the data of daily infections in Pakistan from March 1, 2020 to June 30, 2020, the estimated value of the parameter was obtained. Gupta et al. [40] used a SEIR-QDPA model to simulate COVID-19 transmission in India from March 30, 2020 to June 7, 2020. The best exit time of the blocking strategy is analyzed through experiments. Hezam et al. [41] presented a new mathematical model of cotransmission of COVID19 and cholera, and then fitted the data of coinfection outbreaks in Yemen between 1 January 2020 and 30 May 2020. The study took into account the preventive measures taken to contain the outbreak: social distance, lockdown, number of tests, etc. Wickramaarachchi and Perera [42] used a traditional SEIR model to simulate the spread of COVID-19 in Sri Lanka from 10 March 2020 to 31 May 2020. By assuming that the number of cases of infection presents an exponential curve, the parameters of the obtained data are estimated and the results are analyzed.

It is an important part of infectious disease dynamics to study the dynamic properties of the model. Through the qualitative and quantitative analysis of the model, the development process of the disease is analyzed, the epidemic law is revealed, the change trend is predicted, and the causes and key points of the disease epidemic are analyzed [13-24, 33-35]. For example, Khan et al. [13] established a mathematical model to simulate the spread of Pine Wilt Disease, studied its stability, and compared the control results under different strategies. Li et al. [14] proposed a nonlinear SIQS infectious disease model based on complex networks, and studied the stability of the model by using complex network theory and Lyapunov function method. Bonyah et al. [15] used the Atangana-Beleanu derivative in the sense of Liouville-Caputo to study the model of human African trypanosomiasis, and analyzed the basic properties and stable states in the sense of fractional derivative. Huo et al. [16] considered a new SEIS infectious disease model with media influence, obtained the basic reproduction number through the analysis of the characteristic equation, and studied its stability and the occurrence of Hopf bifurcation. There are also some important articles, please refer to the references.

The basic reproduction number $R_{0}$ is a very important concept in epidemiology, and is undoubtedly "one of the most important and valuable ideas brought by mathematical thinking to the epidemiological theory" [25]. Inspired by the above literatures, in order to demonstrate the impact of Chinese government interventions on COVID-19 transmission, we divide the entire transmission process into three stages according to specific interventions. By fitting daily data published by the National Health Commission, the basic reproduction number for the three stages was obtained. The effect of the intervention was analyzed by comparing the values of the basic reproduction number in three different stages.

The structure of this paper is as follows: the establishment of the model and its basic properties are given in Sect. 2; the basic reproduction number and equilibria are 
obtained in Sect. 3; the stability of the equilibrium are proved in Sect. 4; data analysis and numerical simulation are shown in Sect. 5; some findings of this paper are given in last Section.

\section{The model formulation}

\subsection{System description}

Our research target is the infection of COVID-19 in China, so the target population we selected is all population in China, denoted as $N(t)$. We take the healthy people as $S(t)$. We define people who have close contact with symptomatic and asymptomatic infections as exposed people $E(t)$. They are subject to self-isolation observation.

From the appearance of similar symptoms of COVID-19 to the isolation treatment after diagnosis in the hospital, this is a real process that can not be ignored, because there is still the spread of disease in this process. We define the population in this process as: probable infected population, denoted as $I(t)$. Because asymptomatic infection and mild infection have a lot in common, so we put them into $I_{1}$ compartment. The mild and asymptomatic probable infected individuals are referred to as $I_{1}(t)$; severe probable cases are identified as $I_{2}(t)$. As COVID-19 is a self-limited disease, some people with strong resistance in $I_{1}(t)$ can recover and produce antibodies by relying on their own immunity without entering the hospital for treatment.

Because the COVID-19 is highly contagious, the mild and severe cases are isolated and treated separately to avoid cross-infection among people. Asymptomatic infection once diagnosed in the hospital, will be isolated for treatment. So we generalize this kind of patients to $T_{1}(t)$. Therefore, we divide the confirmed treated patients in the hospital into mild or asymptomatic treatment compartment $T_{1}(t)$ and severe treatment compartment $T_{2}(t)$. People who experienced COVID-19 infection and recovered are referred to as $R(t)$.

Thus, the total population is given by:

$$
N(t)=S(t)+E(t)+I_{1}(t)+I_{2}(t)+T_{1}(t)+T_{2}(t)+R(t) .
$$

The population flow among those compartments is shown in Fig. 1 (Table 1).

The transfer diagram leads to the following system of ordinary differential equations:

$$
\left\{\begin{array}{l}
S^{\prime}=\Lambda-\mu S-S \frac{\alpha_{1} I_{1}+\alpha_{2} I_{2}}{N}, \\
E^{\prime}=S \frac{\alpha_{1} I_{1}+\alpha_{2} I_{2}}{N}-\left(v_{1}+v_{2}+\mu\right) E, \\
I_{1}^{\prime}=v_{1} E-\left(w_{1}+w_{2}+w_{5}+\mu\right) I_{1}, \\
I_{2}^{\prime}=v_{2} E-\left(w_{3}+w_{4}+\mu\right) I_{2}, \\
T_{1}^{\prime}=w_{1} I_{1}+w_{3} I_{2}+\xi T_{2}-\left(\eta_{1}+\eta_{2}+\mu\right) T_{1}, \\
T_{2}^{\prime}=w_{2} I_{1}+w_{4} I_{2}+\eta_{1} T_{1}-(\xi+\mu+d) T_{2}, \\
R^{\prime}=w_{5} I_{1}+\eta_{2} T_{1}-\mu R .
\end{array}\right.
$$




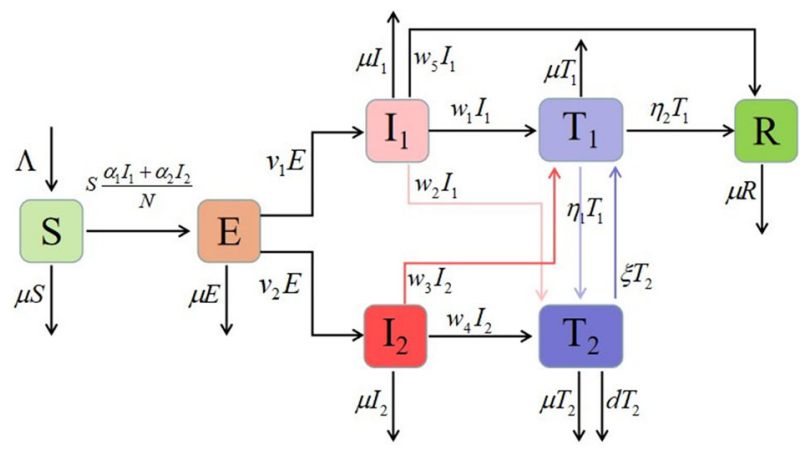

Fig. 1 Transfer diagram of model

\subsection{Positivity}

In practical sense, the initial total number of the model must be positive, and the population of each warehouse is negative at any time. So it is necessary to prove that the solutions of the system (2) with positive initial conditions remain positive for all $t>0$. System (2) can be put into the matrix form

$$
X^{\prime}=G(X)
$$

where $X=\left(S, E, I_{1}, I_{2}, T_{1}, T_{2}, R\right)^{\mathrm{T}} \in R_{7}^{+}$and $\mathrm{G}(\mathrm{X})$ is given by

$$
\begin{aligned}
G(X)= & \left(\begin{array}{l}
G_{1}(X) \\
G_{2}(X) \\
G_{3}(X) \\
G_{4}(X) \\
G_{5}(X) \\
G_{6}(X) \\
G_{7}(X)
\end{array}\right) \\
= & \left(\begin{array}{c}
\Lambda-\mu S-S \frac{\alpha_{1} I_{1}+\alpha_{2} I_{2}}{N} \\
S \frac{\alpha_{1} I_{1}+\alpha_{2} I_{2}}{N}-\left(v_{1}+v_{2}+\mu\right) E \\
v_{1} E-\left(w_{1}+w_{2}+w_{5}+\mu\right) I_{1} \\
v_{2} E-\left(w_{3}+w_{4}+\mu\right) I_{2} \\
w_{1} I_{1}+w_{3} I_{2}+\xi T_{2}-\left(\eta_{1}+\eta_{2}+\mu\right) T_{1} \\
w_{2} I_{1}+w_{4} I_{2}+\eta_{1} T_{1}-(\xi+\mu+d) T_{2} \\
w_{5} I_{1}+\eta_{2} T_{1}-\mu R
\end{array}\right) .
\end{aligned}
$$

We can obtain

$$
\begin{aligned}
& \left.\frac{d S}{d t}\right|_{\left[S=0, E \geq 0, I_{1} \geq 0, I_{2} \geq 0, T_{1} \geq 0, T_{2} \geq 0, R \geq 0\right]}=\Lambda>0, \\
& \left.\frac{d E}{d t}\right|_{\left[S>0, E=0, I_{1} \geq 0, I_{2} \geq 0, T_{1} \geq 0, T_{2} \geq 0, R \geq 0\right]}=S \frac{\alpha_{1} I_{1}+\alpha_{2} I_{2}}{N} \geq 0,
\end{aligned}
$$


Table 1 Description of related parameters in COVID-19 model (2)

\begin{tabular}{|c|c|}
\hline Variables and parameters & Description \\
\hline \multicolumn{2}{|l|}{ Variables } \\
\hline$S(t)$ & The number of health human at time $t$ \\
\hline$E(t)$ & The number of close contacts at time $t$ \\
\hline$I_{1}(t)$ & $\begin{array}{l}\text { The number of asymptomatic and mild symptomatic probable cases with } \\
\text { imaging features of pneumonia }\end{array}$ \\
\hline$I_{2}(t)$ & $\begin{array}{l}\text { The number of severe symptomatic probable cases with imaging features of } \\
\text { pneumonia }\end{array}$ \\
\hline$T_{1}(t)$ & The number of asymptomatic and mild symptomatic patients treated \\
\hline$T_{2}(t)$ & The number of severe symptomatic patients treated \\
\hline$R(t)$ & The number of people recovered \\
\hline \multicolumn{2}{|l|}{ Parameters } \\
\hline$\Lambda$ & The recruitment number of susceptible humans \\
\hline$\mu$ & The natural death rate \\
\hline$\alpha_{1}$ & The effective contact rate of $I_{1}$ \\
\hline$\alpha_{2}$ & The effective contact rate of $I_{2}$ \\
\hline$v_{1}$ & $\begin{array}{l}\text { The rate of disease progression from exposed compartment } E \text { to mild infected } \\
\text { compartment } I_{1}\end{array}$ \\
\hline$v_{2}$ & $\begin{array}{l}\text { The rate of disease progression from exposed compartment } E \text { to severe } \\
\text { infected compartment } I_{2}\end{array}$ \\
\hline$w_{1}$ & $\begin{array}{l}\text { The progression rate from mild infected compartment } I_{1} \text { to mild treatment } \\
\text { compartment } T_{1}(t)\end{array}$ \\
\hline$w_{2}$ & $\begin{array}{l}\text { The progression rate from mild infected compartment } I_{1} \text { to severe treatment } \\
\text { compartment } T_{2}(t)\end{array}$ \\
\hline$w_{3}$ & $\begin{array}{l}\text { The progression rate from severe infected compartment } I_{2} \text { to mild treatment } \\
\text { compartment } T_{1}(t)\end{array}$ \\
\hline$w_{4}$ & $\begin{array}{l}\text { The progression rate from severe infected compartment } I_{2} \text { to severe treatment } \\
\text { compartment } T_{2}(t)\end{array}$ \\
\hline$w_{5}$ & The proportion of self recovery without hospital treatment \\
\hline$\eta_{1}$ & $\begin{array}{l}\text { The progression rate from mild treatment compartment } T_{1} \text { to severe treatment } \\
\text { compartment } T_{2}(t)\end{array}$ \\
\hline$\eta_{2}$ & $\begin{array}{l}\text { The progression rate from severe treatment compartment } T_{2}(t) \text { to mild } \\
\text { treatment compartment } T_{1}\end{array}$ \\
\hline$\xi$ & The proportion of patients recovered from hospital treatment \\
\hline$d$ & The proportion of deaths from COVID-19 \\
\hline
\end{tabular}

$$
\begin{array}{ll}
\left.\frac{d I_{1}}{d t}\right|_{\left[S>0, E \geq 0, I_{1}=0, I_{2} \geq 0, T_{1} \geq 0, T_{2} \geq 0, R \geq 0\right]} & =v_{1} E \geq 0, \\
\left.\frac{d I_{2}}{d t}\right|_{\left[S>0, E \geq 0, I_{1} \geq 0, I_{2}=0, T_{1} \geq 0, T_{2} \geq 0, R \geq 0\right]} & =v_{2} E \geq 0, \\
\left.\frac{d T_{1}}{d t}\right|_{\left[S>0, E \geq 0, I_{1} \geq 0, I_{2} \geq 0, T_{1}=0, T_{2} \geq 0, R \geq 0\right]} & =w_{1} I_{1}+w_{3} I_{2}+\xi T_{2} \geq 0,
\end{array}
$$




$$
\begin{aligned}
& \left.\frac{d T_{2}}{d t}\right|_{\left[S>0, E \geq 0, I_{1} \geq 0, I_{2} \geq 0, T_{1} \geq 0, T_{2}=0, R \geq 0\right]}=w_{2} I_{1}+w_{4} I_{2}+\eta_{1} T_{1} \geq 0, \\
& \left.\frac{d R}{d t}\right|_{\left[S>0, E \geq 0, I_{1} \geq 0, I_{2} \geq 0, T_{1} \geq 0, T_{2} \geq 0, R=0\right]}=w_{5} I_{1}+\eta_{2} T_{1} \geq 0 .
\end{aligned}
$$

Since these rates are non-negative at the boundary of non-negative cone $R_{7}^{+}$, the direction of the vector field is inward from the boundary plane. Therefore, starting from the non-negative point, the trajectories of all solutions remain in the positive region. It is worth noting that some scholars have different solutions to this problem. For more information on this issue, please refer to references [36,37].

\subsection{Boundedness}

Because of

$$
\frac{d N}{d t}=\Sigma_{i=1}^{7} G_{i}(x)=\Lambda-\mu N-d T_{2} \leq \Lambda-\mu N,
$$

which yields that

$$
\limsup _{t \rightarrow \infty} N(t) \leq \frac{\Lambda}{\mu} .
$$

Therefore, we get the biologically feasible region

$$
\Omega=\left\{\left(S, E, I_{1}, I_{2}, T_{1}, T_{2}, R\right) \in R_{+}^{7}: S+E+I_{1}+I_{2}+T_{1}+T_{2}+R \leq \frac{\Lambda}{\mu}\right\},
$$

and it is a positive invariant set of system (2).

\section{The basic reproduction number and existence of endemic equilibrium}

\subsection{The basic reproduction number}

The model has a Disease-Free Equilibrium $D_{0}$ given by

$$
D_{0}=\left(\frac{\Lambda}{\mu}, 0,0,0,0,0,0\right) .
$$

In the following, the basic reproduction number of system (2) will be obtained by the next generation matrix method. Let $x=\left(E, I_{1}, I_{2}, T_{1}, T_{2}, R, S\right)^{\mathrm{T}}$, then system (2) can be written as

$$
\frac{d x}{d t}=\mathscr{F}(x)-\mathscr{V}(x)
$$


where

$$
\mathscr{F}(x)=\left(\begin{array}{c}
S \frac{\alpha_{1} I_{1}+\alpha_{2} I_{2}}{N} \\
v_{1} E \\
v_{2} E \\
0 \\
0 \\
0 \\
0
\end{array}\right), \quad \mathscr{V}(x)=\left(\begin{array}{c}
\left(v_{1}+v_{2}+\mu\right) E \\
\left(w_{1}+w_{2}+w_{5}+\mu\right) I_{1} \\
\left(w_{3}+w_{4}+\mu\right) I_{2} \\
-w_{1} I_{1}-w_{3} I_{2}-\xi T_{2}+\left(\eta_{1}+\eta_{2}+\mu\right) T_{1} \\
-w_{2} I_{1}-w_{4} I_{2}-\eta_{1} T_{1}+(\xi+\mu+d) T_{2} \\
-w_{5} I_{1}-\eta_{2} T_{1}+\mu R \\
\mu S-\Lambda+S \frac{\alpha_{1} I_{1}+\alpha_{2} I_{2}}{N}
\end{array}\right) .
$$

The Jacobian matrices of $\mathscr{F}(x)$ and $\mathscr{V}(x)$ at the Disease-Free Equilibrium $D_{0}$ are

$$
D \mathscr{F}\left(D_{0}\right)=\left(\begin{array}{cc}
F_{5 \times 5} & 0 \\
0 & 0
\end{array}\right), \quad D \mathscr{V}\left(D_{0}\right)=\left(\begin{array}{cc}
V_{5 \times 5} & 0 \\
J_{1} & J_{2}
\end{array}\right)
$$

where

$$
\begin{aligned}
& F_{5 \times 5}=\left(\begin{array}{ccccc}
0 & \alpha_{1} & \alpha_{2} & 0 & 0 \\
v_{1} & 0 & 0 & 0 & 0 \\
v_{2} & 0 & 0 & 0 & 0 \\
0 & 0 & 0 & 0 & 0 \\
0 & 0 & 0 & 0 & 0
\end{array}\right) \\
& V_{5 \times 5}=\left(\begin{array}{ccccc}
v_{1}+v_{2}+\mu & 0 & 0 & 0 & 0 \\
0 & w_{1}+w_{2}+w_{5}+\mu & 0 & 0 & 0 \\
0 & 0 & w_{3}+w_{4}+\mu & 0 & 0 \\
0 & -w_{1} & -w_{3} & \eta_{1}+\eta_{2}+\mu & -\xi \\
0 & -w_{2} & -w_{4} & -\eta_{1} & \xi+\mu+d
\end{array}\right) \text {, } \\
& J_{1}=\left(\begin{array}{ccccc}
0 & -w_{5} & 0 & -\eta_{2} & 0 \\
0 & \alpha_{1} & \alpha_{2} & 0 & 0
\end{array}\right), \quad J_{2}=\left(\begin{array}{ll}
\mu & 0 \\
0 & \mu
\end{array}\right) \text {. }
\end{aligned}
$$

The basic reproduction number, denoted by $R_{0}$, is given by

$$
\begin{aligned}
& R_{0}=\rho\left(F V^{-1}\right)=\sqrt{\frac{1}{v_{1}+v_{2}+\mu}\left(\frac{\alpha_{1} v_{1}}{w_{1}+w_{2}+w_{5}+\mu}+\frac{\alpha_{2} v_{2}}{w_{3}+w_{4}+\mu}\right)} \\
& \triangleq \sqrt{R_{1}}
\end{aligned}
$$

where $\rho(A)$ denotes the spectral radius of a matrix A. 


\subsection{Existence of endemic equilibrium}

The Endemic Equilibrium $D^{*}=\left(S^{*}, E^{*}, I_{1}^{*}, I_{2}^{*}, T_{1}^{*}, T_{2}^{*}, R^{*}\right)$ of system (2) is determined by equations:

$$
\left\{\begin{array}{l}
\Lambda-\mu S-S \frac{\alpha_{1} I_{1}+\alpha_{2} I_{2}}{N}=0 \\
S \frac{\alpha_{1} I_{1}+\alpha_{2} I_{2}}{N}-k_{1} E=0 \\
v_{1} E-k_{2} I_{1}=0 \\
v_{2} E-k_{3} I_{2}=0 \\
w_{1} I_{1}+w_{3} I_{2}+\xi T_{2}-k_{4} T_{1}=0 \\
w_{2} I_{1}+w_{4} I_{2}+\eta_{1} T_{1}-k_{5} T_{2}=0 \\
w_{5} T_{1}+\eta_{2} T_{2}-\mu R=0
\end{array}\right.
$$

where $k_{1}=v_{1}+v_{2}+\mu, k_{2}=w_{1}+w_{2}+w_{5}+\mu, k_{3}=w_{3}+w_{4}+\mu, k_{4}=\eta_{1}+\eta_{2}+\mu$ and $k_{5}=\xi+\mu+d$.

From the first, third and forth equations of the system (9), we get

$$
\begin{aligned}
S & =\frac{\Lambda}{\mu+\left(\frac{\alpha_{1} v_{1}}{N k_{2}}+\frac{\alpha_{2} v_{2}}{N k_{3}}\right) E}, \\
I_{1} & =\frac{v_{1}}{k_{2}} E, \\
I_{2} & =\frac{v_{2}}{k_{3}} E .
\end{aligned}
$$

By adding the first two equations of (9), we have

$$
\Lambda-\mu S=k_{1} E
$$

Hence,

$$
\Lambda-\mu \frac{\Lambda}{\mu+\left(\frac{\alpha_{1} v_{1}}{N k_{2}}+\frac{\alpha_{2} v_{2}}{N k_{3}}\right) E}=k_{1} E
$$

That is

$$
\frac{k_{1}}{\mu N}\left(\frac{\alpha_{1} v_{1}}{k_{2}}+\frac{\alpha_{2} v_{2}}{k_{3}}\right) E^{2}+\left[k_{1}\left(1-\frac{\Lambda}{\mu N} R_{1}\right)\right] E=0 .
$$

Because $N \leq \frac{\Lambda}{\mu}$ and $R_{1}>1,\left[k_{1}\left(1-\frac{\Lambda}{\mu N} R_{1}\right)\right]<0$. From above we know that

$$
E=\frac{1}{k_{1}}\left(\Lambda-\frac{\mu N}{R_{1}}\right)
$$


From the last three equations of (9) we can obtain

$$
\begin{aligned}
& T_{1}=\frac{v_{1} k_{3}\left(k_{5} w_{1}+\xi w_{2}\right)+v_{2} k_{2}\left(k_{5} w_{3}+\xi w_{4}\right)}{k_{2} k_{3}\left(k_{4} k_{5}-\xi \eta_{1}\right)} E, \\
& T_{2}=\frac{v_{1} k_{3}\left(\eta_{1} w_{1}+k_{4} w_{2}\right)+v_{2} k_{2}\left(\eta_{1} w_{3}+k_{4} w_{4}\right)}{k_{2} k_{3}\left(k_{4} k_{5}-\xi \eta_{1}\right)} E \triangleq K E, \\
& R=\frac{v_{1} k_{3}\left[w_{5}\left(k_{5} w_{1}+\xi w_{2}\right)+\eta_{2}\left(\eta_{1} w_{1}+k_{4} w_{2}\right)\right]+v_{2} k_{2}\left[w_{5}\left(k_{5} w_{3}+\xi w_{4}\right)+\eta_{2}\left(\eta_{1} w_{3}+k_{4} w_{4}\right)\right]}{\mu k_{2} k_{3}\left(k_{4} k_{5}-\xi \eta_{1}\right)} E .
\end{aligned}
$$

By adding all equations of (9), we have $\Lambda-\mu N-d T_{2}=0$. Combining Eqs. (10) and (11),

$$
\left(\frac{K d}{k_{1}}-R_{1}\right) \mu N=R_{1} \Lambda\left(\frac{K d}{k_{1}}-1\right)
$$

where

$$
\frac{K d}{k_{1}}=\frac{v_{1} k_{3}\left(\eta_{1} w_{1}+k_{4} w_{2}\right)+v_{2} k_{2}\left(\eta_{1} w_{3}+k_{4} w_{4}\right)}{k_{1} k_{2} k_{3}\left(k_{4} k_{5}-\xi \eta_{1}\right)} d .
$$

Since

$$
\begin{aligned}
d & \left(v_{1} k_{3}\left(\eta_{1} w_{1}+k_{4} w_{2}\right)+v_{2} k_{2}\left(\eta_{1} w_{3}+k_{4} w_{4}\right)\right) \\
& <d\left(v_{1} k_{3}\left(k_{4} w_{1}+k_{4} w_{2}\right)+v_{2} k_{2}\left(k_{4} w_{3}+k_{4} w_{4}\right)\right) \\
& <d\left(v_{1} k_{3} k_{4} k_{2}+v_{2} k_{2} k_{4} k_{3}\right) \\
& <d k_{1} k_{2} k_{3} k_{4} \\
& <d k_{1} k_{2} k_{3} k_{4}+\xi k_{1} k_{2} k_{3}\left(k_{4}-\eta_{1}\right) \\
& <k_{1} k_{2} k_{3} k_{4} k_{5}-k_{1} k_{2} k_{3} \xi \eta_{1} .
\end{aligned}
$$

Thus, $0<K d / k_{1}<1$, we can get that the value of $N$ is unique and positive as $R_{1}>1$. So there is a unique positive Endemic Equilibrium $D^{*}$ when $R_{0}>1$.

Theorem 1 In the system (2), there is always a Disease-Free Equilibrium $D_{0}=$ $\left(\frac{\Lambda}{\mu}, 0,0,0,0,0,0\right)$. When $R_{0}>1$, the system (2) has a unique Endemic Equilibrium $D^{*}=\left(S^{*}, E^{*}, I_{1}^{*}, I_{2}^{*}, T_{1}^{*}, T_{2}^{*}, R^{*}\right)$. 


\section{Stability analysis of disease-free equilibrium}

We denote a vector $x=\left(E, I_{1}, I_{2}, T_{1}, T_{2}, R, S\right)^{\mathrm{T}}$ and

$$
f(x)=\left(\begin{array}{c}
S \frac{\alpha_{1} I_{1}+\alpha_{2} I_{2}}{N}-\left(v_{1}+v_{2}+\mu\right) E \\
v_{1} E-\left(w_{1}+w_{2}+w_{5}+\mu\right) I_{1} \\
v_{2} E-\left(w_{3}+w_{4}+\mu\right) I_{2} \\
w_{1} I_{1}+w_{3} I_{2}+\xi T_{2}-\left(\eta_{1}+\eta_{2}+\mu\right) T_{1} \\
w_{2} I_{1}+w_{4} I_{2}+\eta_{1} T_{1}-(\xi+\mu+d) T_{2} \\
w_{5} I_{1}+\eta_{2} T_{1}-\mu R \\
\Lambda-\mu S-S \frac{\alpha_{1} I_{1}+\alpha_{2} I_{2}}{N}
\end{array}\right) .
$$

So the Jacobian matrix of $f(x)$ about vector $x$ is as the following:

$$
\begin{aligned}
J & =\frac{\partial f(x)}{\partial x} \\
& =\left(\begin{array}{ccccccc}
-k_{1} & \alpha_{1} \frac{S}{N} & \alpha_{2} \frac{S}{N} & 0 & 0 & 0 & \frac{\alpha_{1} I_{1}+\alpha_{2} I_{2}}{N} \\
v_{1} & -k_{2} & 0 & 0 & 0 & 0 & 0 \\
v_{2} & 0 & -k_{3} & 0 & 0 & 0 & 0 \\
0 & w_{1} & w_{3} & -k_{4} & 0 & 0 & 0 \\
0 & w_{2} & w_{4} & 0 & -k_{5} & 0 & 0 \\
0 & 0 & 0 & \eta & \xi & -\mu & 0 \\
0 & -\alpha_{1} \frac{S}{N} & -\alpha_{2} \frac{S}{N} & 0 & 0 & 0 & -\frac{\alpha_{1} I_{1}+\alpha_{2} I_{2}}{N}-\mu
\end{array}\right) .
\end{aligned}
$$

where $k_{1}=v_{1}+v_{2}+\mu, k_{2}=w_{1}+w_{2}+w_{5}+\mu, k_{3}=w_{3}+w_{4}+\mu, k_{4}=\eta_{1}+\eta_{2}+\mu$ and $k_{5}=\xi+\mu+d$.

Theorem 2 For the system (2), the Disease-Free Equilibrium $D_{0}$ is Locally Asymptotically Stable $(L A S)$ if $R_{0}<1$.

Proof Since

$$
J\left(D_{0}\right)=\left(\begin{array}{ccccccc}
-k_{1} & \alpha_{1} & \alpha_{2} & 0 & 0 & 0 & 0 \\
v_{1} & -k_{2} & 0 & 0 & 0 & 0 & 0 \\
v_{2} & 0 & -k_{3} & 0 & 0 & 0 & 0 \\
0 & w_{1} & w_{3} & -k_{4} & \xi & 0 & 0 \\
0 & w_{2} & w_{4} & \eta_{1} & -k_{5} & 0 & 0 \\
0 & w_{5} & 0 & \eta_{2} & 0 & -\mu & 0 \\
0 & -\alpha_{1} & -\alpha_{2} & 0 & 0 & 0 & -\mu
\end{array}\right)
$$

We assume that $\lambda_{1}, \lambda_{2}, \lambda_{3}$ are eigenvalues of $M_{1}, \lambda_{4}, \lambda_{5}$ are eigenvalues of $M_{2}$ and $\lambda_{6}=\lambda_{7}=-\mu$, where

$$
M_{1}=\left(\begin{array}{ccc}
-k_{1} & \alpha_{1} & \alpha_{2} \\
v_{1} & -k_{2} & 0 \\
v_{2} & 0 & -k_{3}
\end{array}\right), \quad M_{2}=\left(\begin{array}{cc}
-k_{4} & \xi \\
\eta_{1} & -k_{5}
\end{array}\right)
$$


The characteristic equation of characteristic matrix of $M_{1}$ is

$$
\lambda^{3}+b_{1} \lambda^{2}+b_{2} \lambda+b_{3}=0
$$

where

$$
\begin{aligned}
& b_{1}=k_{1}+k_{2}+k_{3}, \\
& b_{2}=k_{2} k_{3}+k_{1} k_{2}+k_{1} k_{3}-\alpha_{2} v_{2}-\alpha_{1} v_{1}, \\
& b_{3}=k_{1} k_{2} k_{3}-\alpha_{2} v_{2} k_{2}-\alpha_{1} v_{1} k_{3} .
\end{aligned}
$$

Due to $0<R_{0}<1$, we have $b_{1}>0, b_{3}>0, \alpha_{1} v_{1}<k_{1} k_{2}$ and $\alpha_{2} v_{2}<k_{1} k_{3}$.

$$
\begin{aligned}
& b_{1} b_{2}-b_{3} \\
& =\left(k_{1}+k_{2}+k_{3}\right)\left(k_{2} k_{3}+k_{1} k_{2}+k_{1} k_{3}-\alpha_{2} v_{2}-\alpha_{1} v_{1}\right)-\left(k_{1} k_{2} k_{3}-\alpha_{2} v_{2} k_{2}-\alpha_{1} v_{1} k_{3}\right) \\
& =k_{1}\left(k_{1} k_{2}+k_{1} k_{3}-\alpha_{2} v_{2}-\alpha_{1} v_{1}\right)+\left(k_{2}+k_{3}\right)\left(k_{2} k_{3}+k_{1} k_{2}+k_{1} k_{3}-\alpha_{2} v_{2}-\alpha_{1} v_{1}\right) \\
& \quad+\left(k_{2} \alpha_{2} v_{2}+k_{3} \alpha_{1} v_{1}\right) \\
& >0 .
\end{aligned}
$$

Using Routh-Hurwitz criteria, the real part of all characteristic roots of $M_{1}$ are negative. The characteristic equation of characteristic matrix of $M_{2}$ is

$$
\lambda^{2}+\left(\eta_{1}+\eta_{2}+2 \mu+\xi+d\right) \lambda+\eta_{1}(\mu+d)+\left(\eta_{2}+\mu\right)(\xi+\mu+d)=0 .
$$

Because all coefficients of the equation are greater than 0 , the real part of all characteristic roots of $M_{2}$ are negative. So the point $D_{0}$ is LAS. The proof is completed.

Theorem 3 For the system (2), the Disease-Free Equilibrium $D_{0}$ is Globally Asymptotically Stable $(G A S)$ if $R_{0}<1$.

Proof Consider the subsystem of (2) as follows:

$$
\begin{aligned}
& E^{\prime}(t)=S \frac{\alpha_{1} I_{1}+\alpha_{2} I_{2}}{N}-k_{1} E, \\
& I_{1}^{\prime}(t)=v_{1} E-k_{2} I_{1}, \\
& I_{2}^{\prime}(t)=v_{2} E-k_{3} I_{2}, \\
& T_{1}^{\prime}(t)=w_{1} I_{1}+w_{3} I_{2}+\xi T_{2}-k_{4} T_{1}, \\
& T_{2}^{\prime}(t)=w_{2} I_{1}+w_{4} I_{2}+\eta_{1} T_{1}-k_{5} T_{2} .
\end{aligned}
$$

For $S \leq N \quad($ all $\mathrm{t}>0)$,

$$
\left(\begin{array}{c}
E^{\prime} \\
I_{1}^{\prime} \\
I_{2}^{\prime} \\
T_{1}^{\prime} \\
T_{2}^{\prime}
\end{array}\right) \leq\left(\begin{array}{c}
\alpha_{1} I_{1}+\alpha_{2} I_{2}-k_{1} E \\
v_{1} E-k_{2} I_{1} \\
v_{2} E-k_{3} I_{2} \\
w_{1} I_{1}+w_{3} I_{2}+\xi T_{2}-k_{4} T_{1} \\
w_{2} I_{1}+w_{4} I_{2}+\eta_{1} T_{1}-k_{5} T_{2}
\end{array}\right)
$$




$$
=(F-V)\left(\begin{array}{c}
E \\
I_{1} \\
I_{2} \\
T_{1} \\
T_{2}
\end{array}\right) .
$$

Since the eigenvalues of the matrix $F-V$ all have negative real parts, then system (2) is stable when $R_{0}<1$. So $\left(E, I_{1}, I_{2}, T_{1}, T_{2}\right) \rightarrow(0,0,0,0,0)$ as $t \rightarrow \infty$. By the comparison theorem, it follows that $\left(E, I_{1}, I_{2}, T_{1}, T_{2}\right) \rightarrow(0,0,0,0,0)$ and $S \rightarrow \frac{\Lambda}{\mu}$ as $t \rightarrow \infty$. So $D_{0}$ is Globally Asymptotically Stable for $R_{0}<1$.

Remark 1 For more information on the global stability results, please refer to reference $[37,38]$.

Remark 2 It is worth mentioning that the unique Endemic Equilibrium $D^{*}$ is unstable from the literature [30] and the trend prediction analysis part of the first stage in numerical simulation. If we set that the death rate $d$ due to COVID-19 as 0, then we can prove that the Endemic Equilibrium is GAS. However, it is obvious that this assumption is meaningless because it is seriously inconsistent with the actual situation.

\section{Numerical simulation}

In this chapter, we simulate the number of probable, mild, severe and recovered patients reported by the National Health Commission in different stages, and estimate the parameters by using the nonlinear weighted least square method to get the value of the basic reproduction number, so as to explore the spread of disease in this stage and the role of the government's strong intervention policy.

\subsection{Data collection}

The data of COVID-19 in China from January 10, 2020 to March 11, 2020 are collected from the web of the National Health Commission, including number of the probable, confirmed and recovered cases, see attachment 1 for more details. Figure 2a shows the new confirmed cases per day, and Fig. $2 b$ shows the cumulative confirmed cases per day. It can be seen from Fig. 2 that the curves of the number of new cases and the cumulative number of cases every day are relatively smooth, except for two abnormal points: 1771 new confirmed cases and 4515 cumulative confirmed cases on January 27, 15,152 new cases and 59,804 cumulative confirmed cases on February 12.

The reason for the first abnormal point of statistical data is that at the beginning of the outbreak of the COVID-19, people don't know much about the virus, such as its transmission route, transmission intensity, and whether there will be human to human phenomenon. On January 26, almost all cities in China implemented measures of isolation at home. Therefore, we will take the period from January 10 to January 26 as the first stage of disease transmission. In the first stage, the disease is transmitted naturally without people's awareness of protection. At the same time, there may be a certain gap between the statistical data of this stage and the real infection situation. 
(a)
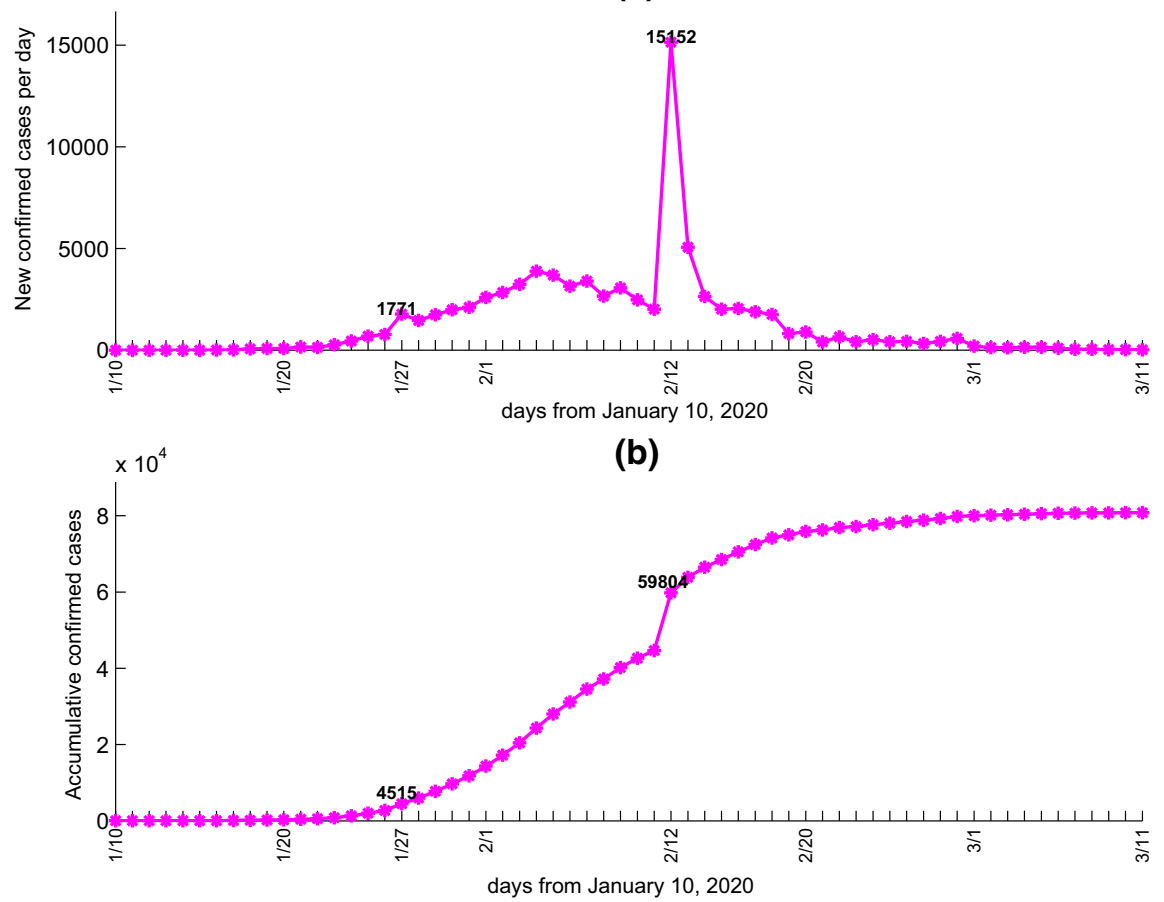

Fig. 2 The number of new confirmed cases and the cumulative confirmed cases per day in China from January 10, 2020 to March 11, 2020: (a, b)

From January 27 to February 11, people consciously cut off all social activities, greatly reducing the chance of virus transmission. As the incubation period of COVID19 is 14 days, with an average of 7 days, in Fig. 2a, we can see that there is still a continuous increase in new cases every day for 8 consecutive days, and then the decrease begins. Because the detection method of COVID-19 is still under study, there are still many suspected cases not detected in this period of time. Until around February 11th, the detection reagent of the COVID-19 was produced on a large scale, and the number of confirmed statistics had an abnormal surge. Therefore, we regard the spread of the disease between January 27 and February 11 as the second stage. In this stage, people have a strong sense of self-protection, and under the call of the government, they actively isolate themselves at home. Due to the large-scale production of COVID-19 detection reagents, huoshenshan and leishenshan anti epidemic hospital and fangcang hospital were built in this period of time. Therefore, during this period, the total number of suspected and confirmed patients is very close to the total number of real infected patients, and the data is relatively reliable.

The reason for the second abnormal point in the statistical data is that on February 12 , the new coronavirus detection reagent was fully supplied, and the existing suspected cases were tested and diagnosed in a wide range, so an explosive growth abnormal point will appear in the new cases. From February 12 onwards, we can see 
from Fig. 2a that the number of new cases continues to decrease, indicating that the spread of the virus is significantly reduced. All these show that the strong intervention of the Chinese government has played a very effective role in curbing the spread of the virus.

\subsection{Parameter estimation}

First of all, we estimate some parameters through the existing literature. In 2020, the population statistics report pointed out that the average life expectancy of Chinese is 77 years old [26]. Therefore, we have $\mu=1 /(77 * 365)$ per day. According to the population statistics report, the total number of Chinese mainland is $1,400,050,000$ [26], so $\Lambda=49,814.98$ per day. We use the cumulative number of deaths reported per day divided by the cumulative number of confirmed cases, and then take its mean value as the estimation of mortality $d$. The death rates of the three stages are: $d_{1}=0.028398$, $d_{2}=0.021824, d_{3}=0.032834$. According to the objective situation, the treatment level in the third stage should be higher than that in the first and second stages, so the mortality $d_{3}$ should be less than $d_{1}$ and $d_{2}$. This inconsistency with reality may be caused by incomplete data collection in the first and second stages.

In this estimation method, the data is fitted by minimizing the weighted error square sum between the estimated values and the reported data. We take the data of probable infection cases, mild confirmed cases, severe confirmed cases and cure cases reported as the target of $I_{1}+I_{2}, T_{1}, T_{2}, R$ to fit. Let's set the objective function in the minimization process as follows.

$$
\begin{aligned}
J= & W_{1} \sum_{i=1}^{n}\left[\left(I_{1}+I_{2}\right)_{t_{i}}-(\tilde{I})_{t_{i}}\right]^{2}+W_{2} \sum_{i=1}^{n}\left[\left(T_{1}\right)_{t_{i}}-\left(\tilde{T}_{1}\right)_{t_{i}}\right]^{2} \\
& +W_{3} \sum_{i=1}^{n}\left[\left(T_{2}\right)_{t_{i}}-\left(\tilde{T}_{2}\right)_{t_{i}}\right]^{2} \\
& +W_{4} \sum_{i=1}^{n}\left[(R)_{t_{i}}-(\tilde{R})_{t_{i}}\right]^{2}
\end{aligned}
$$

where $I_{1}+I_{2}$ is the estimated number of probable cases; $T_{1}, T_{2}, R$ is the estimated number of mild treatment, severe treatment and recovered cases; $\tilde{I}, \tilde{T}_{1}, \tilde{T}_{2}, \tilde{R}$ is the reported number of probable, mild treatment, severe treatment and recovered cases; $W_{i},(i=1,2,3,4)$ is the weight coefficient of the error square sum in each compartment, and the value of $W_{i}$ is the reciprocal of the sample point variance. The selection of weight can effectively reduce the fitting deviation caused by the difference in the order of magnitude of different warehouses' data. There are also some introductions about this method, please refer to [27,28]. To estimate the parameters of the model, we need to minimize the following objective function.

$$
\left\{\begin{array}{l}
\min J \\
\text { subject to system (2). }
\end{array}\right.
$$


(a)

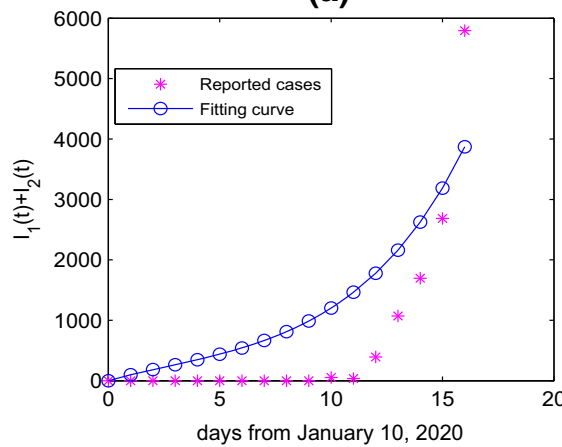

(c)

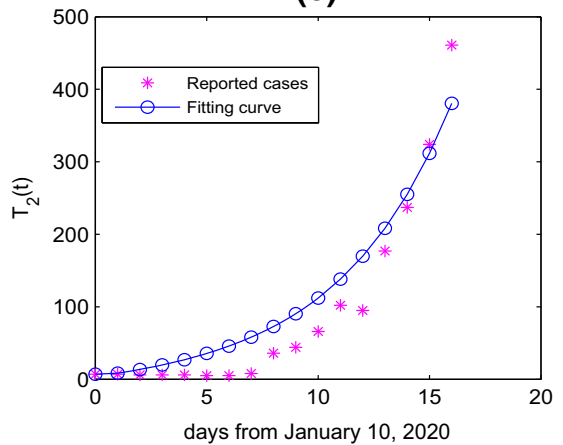

(b)

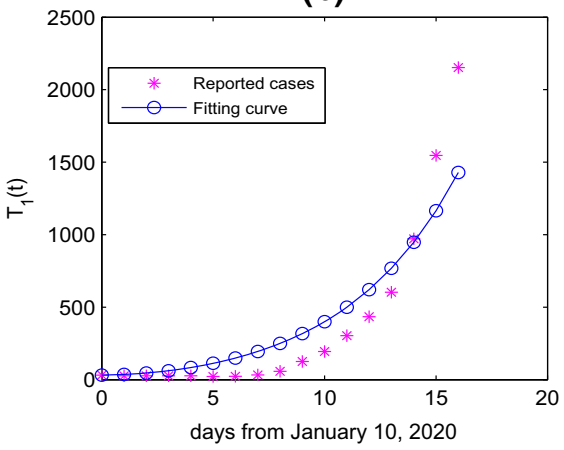

(d)

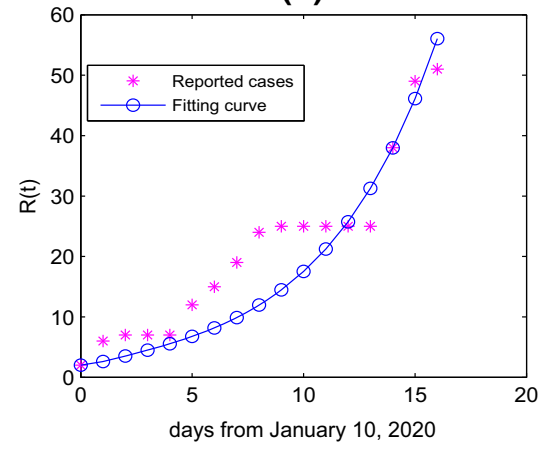

Fig. 3 Model fitting for the reported number of probable cases, mild treatment cases, severe treatment cases and recovered cases in the first stage (January 10, 2020 to January 26, 2020)

In the first stage, the fitting results of probable cases, mild treatment cases, severe treatment cases and recovered cases are shown in Fig. 3a-d respectively. The results of the fitted parameters are shown in Table 2.

In Fig. 3a-c, we can see a common feature: the simulated values are larger than the reported values. This is consistent with the previous analysis. In the early stage of disease outbreak, the lack of understanding of the disease led to incomplete statistical data. Therefore, there are some differences between the fitted results and the reported data. From the fitting results, the virus outbreak shows an exponential growth trend, so its transmission speed is very fast. By substituting the fitting parameters into $R_{0}$, we get that the basic reproduction number of the COVID-19 in the first stage (natural state) is $R_{01}=2.6735$. This result is close to the simulation result 2.4829 in [7].

In the second stage, under the strict measures of the government, almost all cases have been counted, so the sum of the probable cases and confirmed cases is very close to the number of real infections, and the number of severe cases has been basically treated in the hospital, so the data is reliable. The simulation results are shown in Fig. 4.

In Fig. 4a-d, the number of probable, mild treatment, severe treatment, and severe cases per day during the second stage is shown. At this stage, we can see that although 


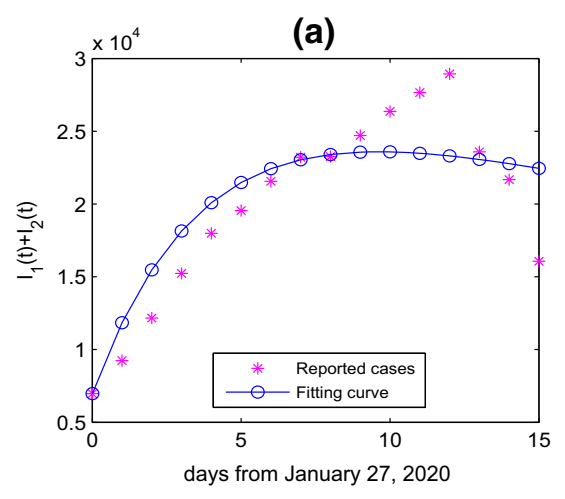

(c)

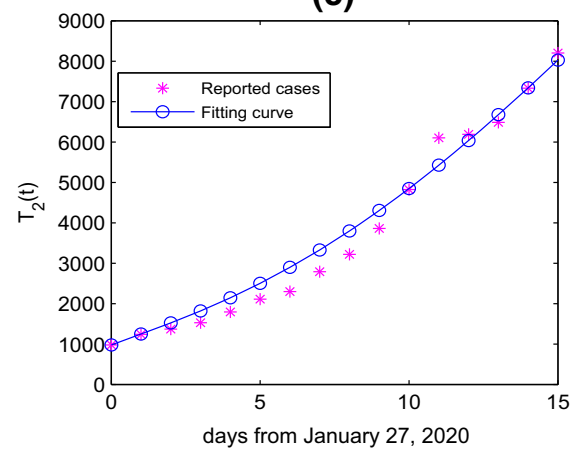

(b)

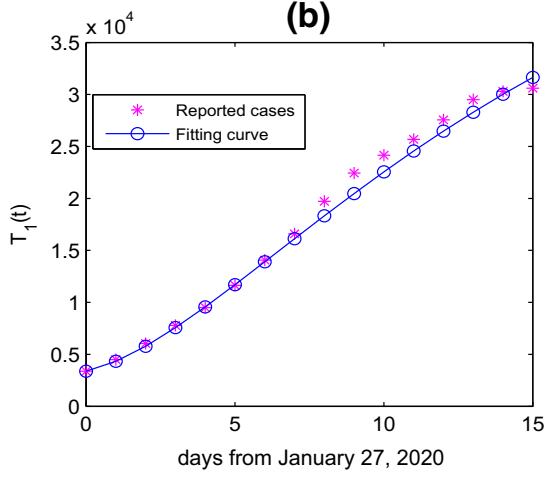

(d)

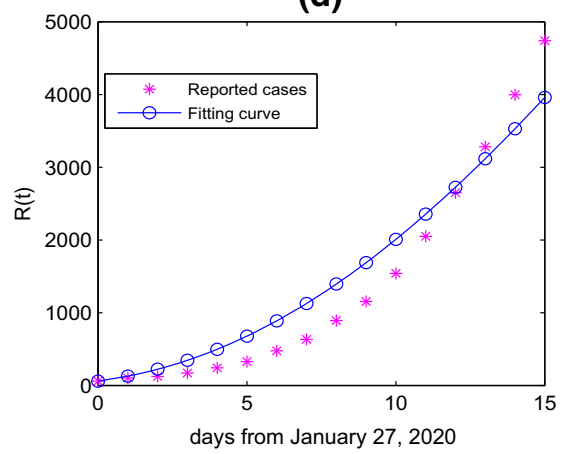

Fig. 4 Model fitting for the reported number of probable cases, mild treatment cases, severe treatment cases and recovered cases in the second stage (January 27, 2020 to February 11, 2020)

the number of confirmed cases has been increasing, the rate of increase has been gradually decreasing, indicating that the spread of the virus has been effectively suppressed. We substituted the fitted parameters into $R_{0}$ and obtained: $R_{02}=0.85077<1$ for the second stage (isolated state) transmission of the COVID-19. From a biological point of view, when the basic reproduction number is less than 1, after a certain period of time, the virus will eventually disappear. When people persist in isolation for a certain period of time, blocking the spread of the virus, the disease will disappear.

Figure 5a-d shows the number of probable, mild treatment, severe treatment and recovered cases per day during the third stage. In this stage, we can see that the number of cases in any subgraph dropped sharply after a few days, which also indicates that the combination of isolation, detection and treatment can quickly and effectively inhibit the spread of the virus. The actual number of patients in the mild and severe treatment compartments are lower than that in the simulation, indicating that the effect of treatment is beyond expectation and satisfactory.

We substituted the fitted parameters into $R_{0}$ and obtained that the basic reproduction number of the COVID-19 in the third stage (isolation + detection + treatment state) transmission $R_{03}=0.18249$. It is far less than 1 , so there is a sharp drop in the number 
(a)

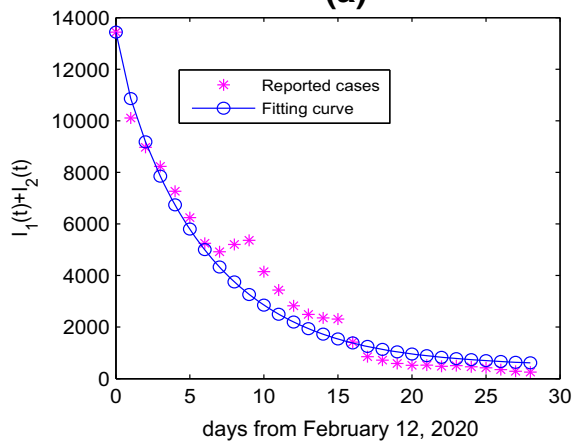

(c)

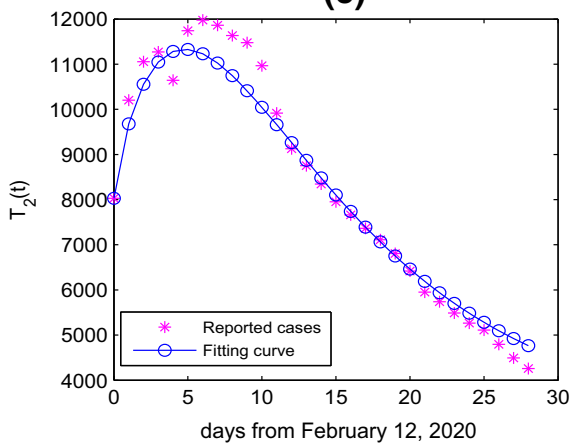

(b)

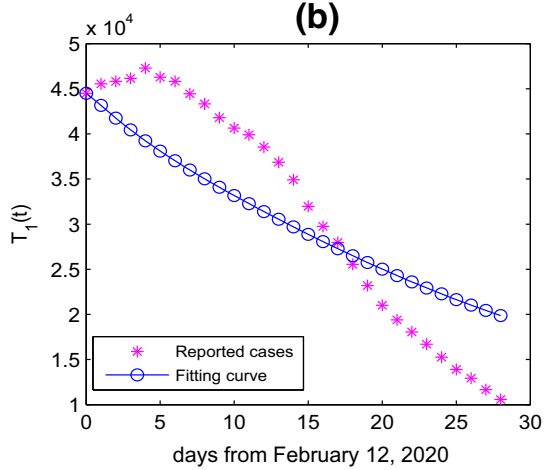

(d)

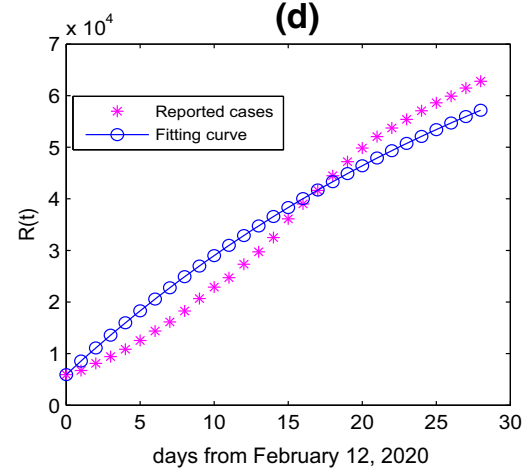

Fig. 5 Model fitting for the reported number of probable cases, mild treatment cases, severe treatment cases and recovered cases in the third stage (February 12, 2020 to March 11, 2020)

of people infected in the third stage, and it also shows that the anti-virus measures implemented in China are very effective.

\subsection{Sensitivity analysis}

In order to further study the leading factors related to an intervention and having a significant impact on the disease dynamics, we need to conduct sensitivity analysis (SA) on the model. For this reason, we use the standard combination of Latin hypercube sampling (LHS) and partial rank correlation coefficient (PRCC). PRCC can be successfully used to measure the monotonic nonlinear relationship between the input and output of the system. This is one of the most effective statistical techniques. For more information about this method, please refer to [31,32].

In Figs. 6, 7 and 8, we depict the results of PRCC graphically in three different stages. To generate the LHS matrix, we assume that all model parameters are uniformly distributed. The model was simulated for 1000 times in each stage. .

We can see that the parameters with high and positive PRCC values are as follows: $\alpha_{2}, w_{2}$ and $\xi$ in Fig. 6, $\alpha_{2}, v_{1}$ and $v_{2}$ in Fig. 7, $\alpha_{2}, v_{1}$ and $\xi$ in Fig. 8. This means that we can reduce the basic reproduction number $R_{0}$ by reducing the values of these 
Table 2 Fitted or assumed values of parameters for system (2) (unit: day ${ }^{-1}$ )

\begin{tabular}{lllll}
\hline Parameters & Stage 1 & Stage 2 & Stage 3 & Source \\
\hline$\Lambda$ & $49,814.98$ & $49,814.98$ & $49,814.98$ & Assumed \\
$\mu$ & $1 /(77 * 365)$ & $1 /(77 * 365)$ & $1 /(77 * 365)$ & {$[26]$} \\
$\alpha_{1}$ & 0.103652264165970 & 0.0934478777510194 & 0.015732621270915 & Fitted \\
$\alpha_{2}$ & 0.673804018259787 & 0.073271050672040 & 0.0523709944899440 & Fitted \\
$v_{1}$ & 0.005466677784469 & 0.146368512043268 & 0.000126932635995 & Fitted \\
$v_{2}$ & 0.146785395488717 & 0.003270082168560 & 0.003631626073494 & Fitted \\
$w_{1}$ & 0.866167996386389 & 0.124560244196773 & 0.000145574031825 & Fitted \\
$w_{2}$ & 0.858826422041044 & 0.000100508226297 & 0.163487012708329 & Fitted \\
$w_{3}$ & 0.026843195666970 & 0.000503074786105 & 0.741960901438499 & Fitted \\
$w_{4}$ & 0.064005285176012 & 0.295893023728773 & 0.924183989377603 & Fitted \\
$w_{5}$ & 0.572206716292525 & 0.002506880303582 & 0.000008218253191 & Fitted \\
$\eta_{1}$ & 0.142046442650272 & 0.026922718755618 & 0.003119264595919 & Fitted \\
$\eta_{2}$ & 0.001059046324261 & 0.012200231351765 & 0.060211095648758 & Fitted \\
$\xi$ & 0.991034934244199 & 0.005530790005271 & 0.090562306732993 & Fitted \\
$d$ & 0.028398 & 0.021824 & 0.032834 & Assumed \\
\hline
\end{tabular}

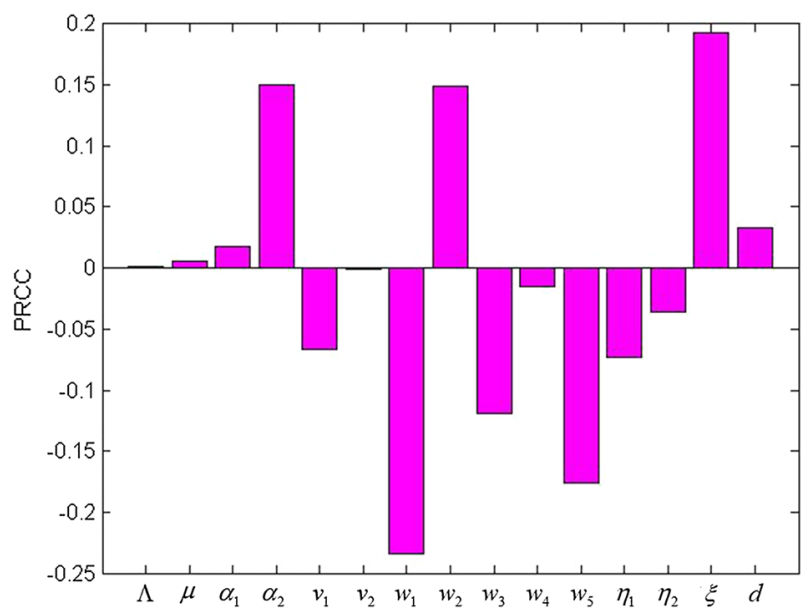

Fig. 6 PRCC values for the COVID-19 model (2) in the first stage

parameters. At the same time, we can found that the parameters with high and negative PRCC values are as follows: $w_{1}, w_{3}$ and $w_{5}$ in Fig. 6, $w_{1}, w_{2}$ and $w_{5}$ in Fig. 7, $w_{1}, w_{3}$ and $w_{5}$ in Fig. 8. This also means that in different stages, we can reduce the value of $R_{0}$ by increasing the value of these parameters. These results reflect the contribution of different parameters to the system dynamics in each stage. We can use these data to guide the daily epidemic prevention work. 


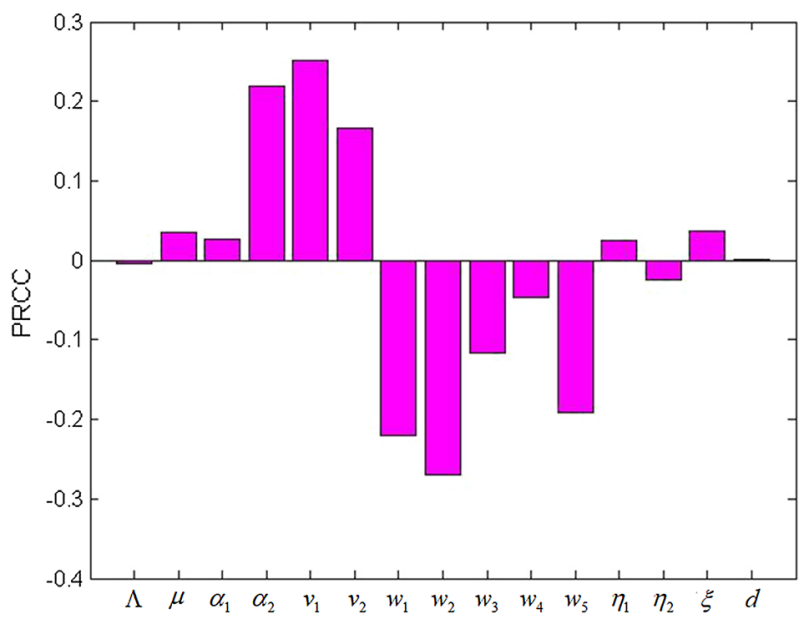

Fig. 7 PRCC values for the COVID-19 model (2) in the second stage

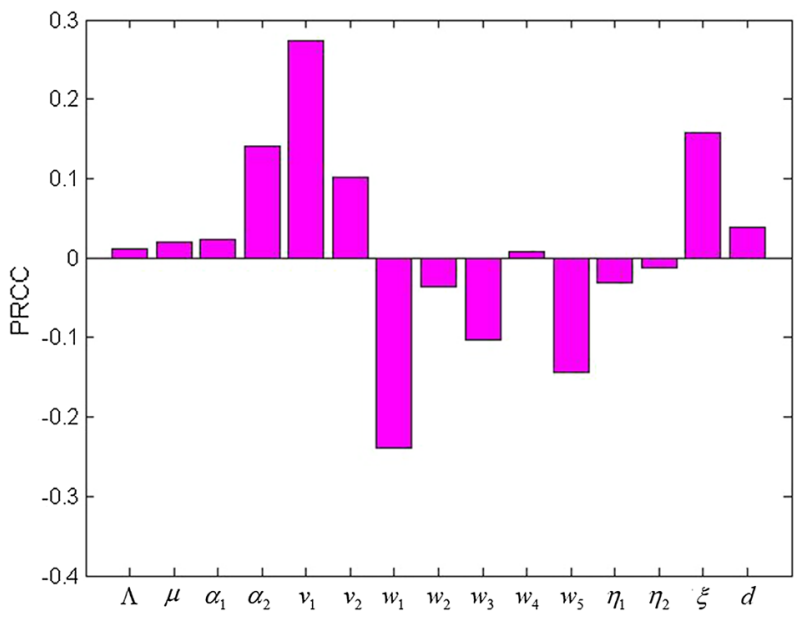

Fig. 8 PRCC values for the COVID-19 model (2) in the third stage

\subsection{Prediction analysis}

We continue to use the previously fitted parameters in the first stage for forward simulation and prediction, and the results are shown in Fig. 9. The blue line represent the population changes of each warehouse under the natural propagation conditions. Since the basic number of regenerations at this stage is $R_{01}=2.6735$, the number of confirmed cases will reach the peak after about 100 days. Under this assumption, the number of confirmed cases remains at 1.1 billion, the number of mild cases treated at 980 million, and the number of severe cases at 141 million. This is really an unimaginable and unacceptable result. 

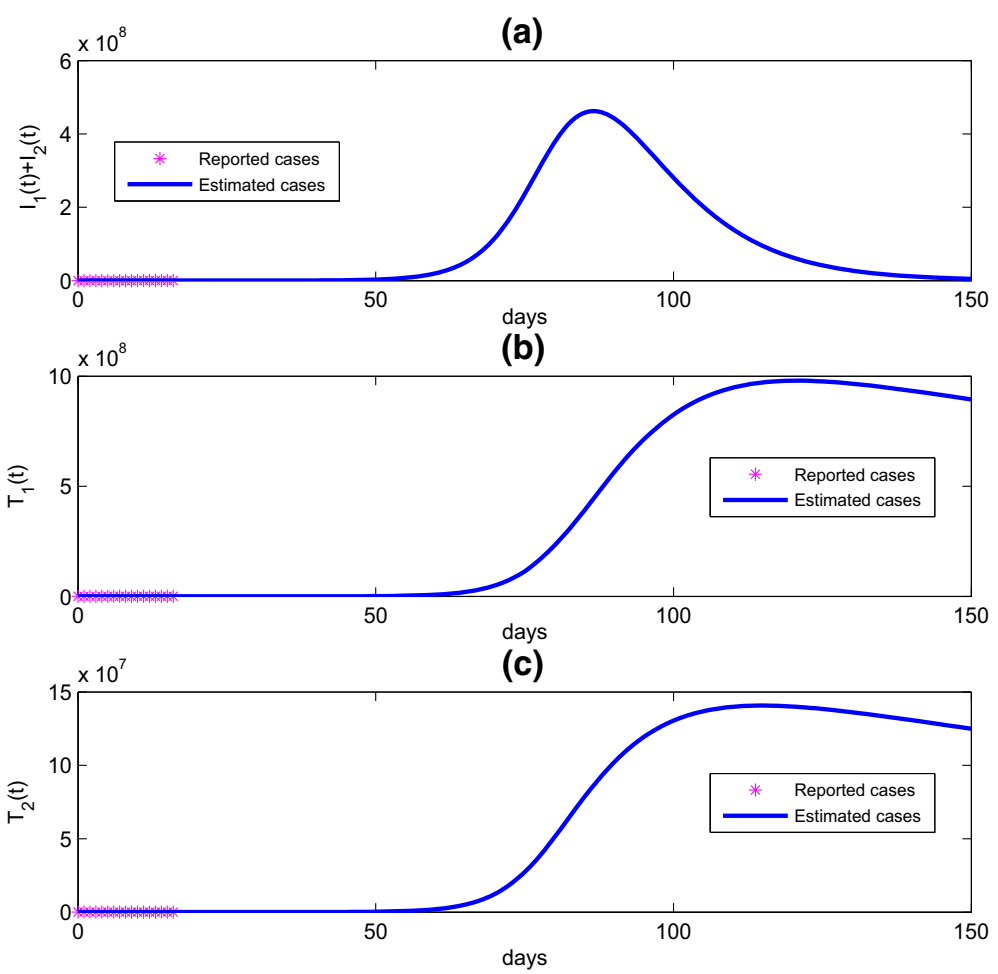

Fig. 9 Population change curve of probable, mild treatment and severe treatment cases in the first stage

The fitted parameter values of the second stage and the third stage are used to predict the results, which are shown in Figs. 10 and 11. As you can see from the graph, they all converge to DFE $D_{0}$. Since their basic reproduction numbers are all less than 1 , they also tend to be in a stable state, which is consistent with the global stability of DFE $D_{0}$ in our previous theory.

As the prevention and control measures in the second stage are still based on isolation, there are some differences between the second and third stage. In Figs. 10 and 11 , we can see from the comparison results that the number of confirmed cases in the third stage will decline faster. It indicates that the treatment scheme of combining traditional Chinese and Western medicine in the hospital plays a very important role in the recovery of patients. At the same time, it can also be used as a basis for other countries to learn from the treatment scheme of combining traditional Chinese and Western medicine in the fight against the epidemic.

\section{Conclusion}

In early 2020, the spread of COVID-19 in mainland China posed a serious threat to people's lives. The resolute measures taken by the Chinese government have brought 

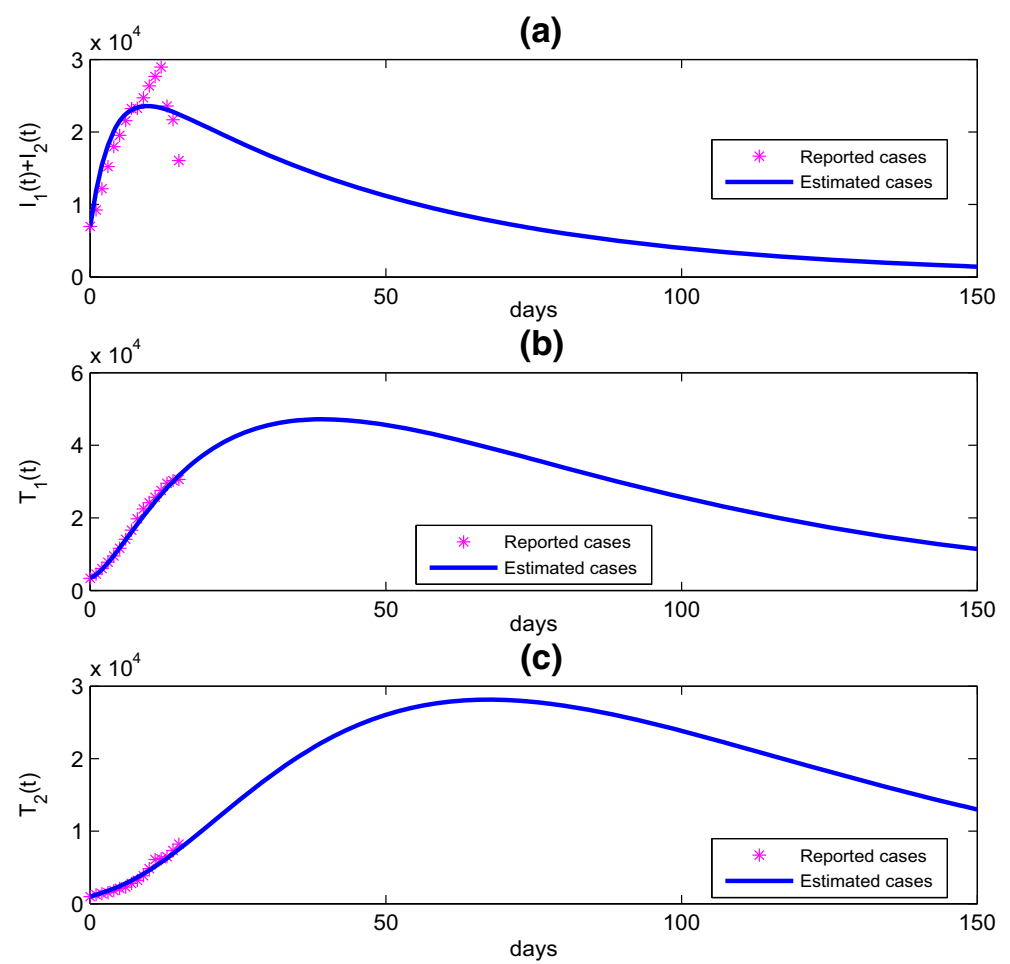

Fig. 10 Population change curve of probable, mild treatment and severe treatment cases in the second stage

the spread of the disease under rapid and effective control. In this study, a novel mathematical model of COVID-19 was established to simulate the spread of COVID19 in China.

In the analysis of the dynamic properties of the model, the basic properties (positivity and boundedness) of the solution of the model are firstly proved, and then the existence of all equilibria of the model are obtained. The basic reproduction number $R_{0}$ of the model is obtained by the classical method of next generation matrix. Finally, the stability of all equilibria are analyzed.

In the numerical simulation, we first divided the entire COVID-19 transmission process into three stages, based on data from the National Health Commission website and strong national interventions. Then, the nonlinear weighted least square estimation method is used to fit the data of multiple warehouses, and the parameter estimation values of each stage are obtained, and the basic reproduction numbers of three different stages are calculated. Then the global sensitivity analysis of the threshold parameters at each stage were carried out to obtain the parameters that had a greater influence on the disease transmission. Finally, through the trend analysis of each stage, we got their change trend. Based on the basic reproduction numbers and trend changes in the three stages, we can see that the intervention has played a very important role in inhibiting the transmission of COVID-19. 
(a)
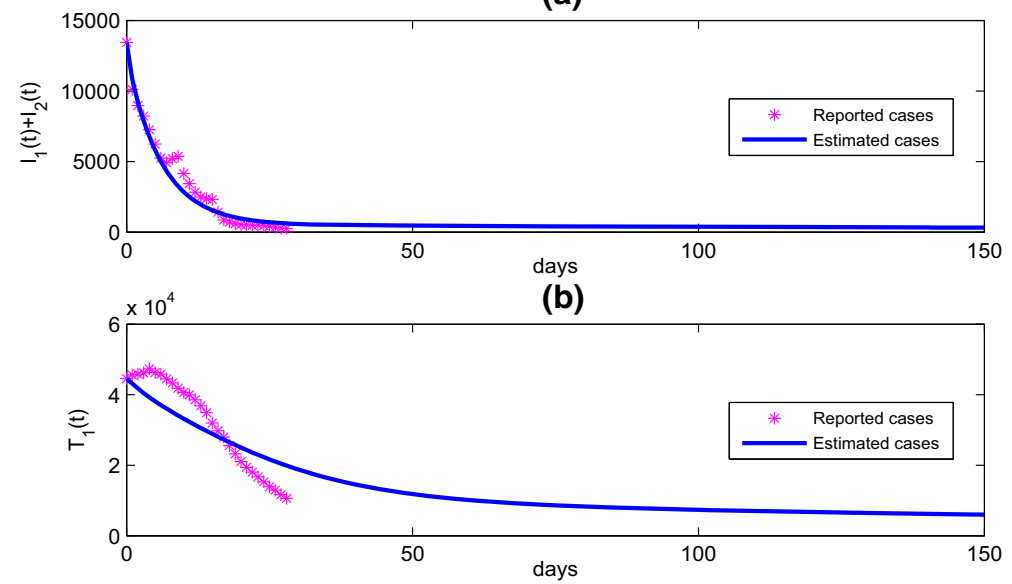

(c)

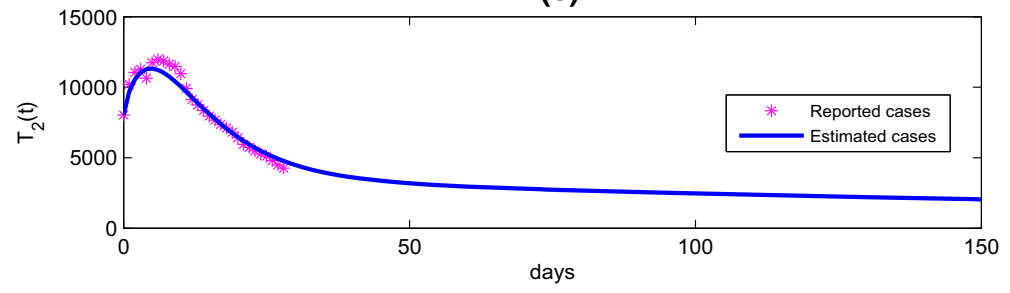

Fig. 11 Population change curve of probable, mild treatment and severe treatment cases in the third stage

The differences between this paper and previous literatures lie in the following points:

(i) Differences in data processing. Considering that government intervention measures will change people's behavior, will greatly affect the spread rate of the disease, and will also greatly change the values of various parameters. The numerical simulation results prove our point of view. Therefore, we divided the whole process into three stages for analysis and comparison according to specific measures, which is an important difference between this paper and many COVID-19 papers.

(ii) Differences in COVID-19 model. Based on the traditional SEIR model, in this paper we further considered that asymptomatic infected persons are also infectious, and that mild and severe cases are treated separately in hospital.

(iii) Differences in data fitting methods. In this paper, in the process of data fitting, in order to fully use the data, consider using the nonlinear weighted least square estimation method to fit the data of multiple warehouses. This point is different from many articles that only fit the cases of infected compartment. So the results we get are relatively more reliable.

In the numerical simulation, we analyzed the global sensitivity analysis of the lower threshold parameters at each stage, and obtained some parameters that have a greater influence on the spread of the disease. Combined with the actual epidemic prevention 
and control measures, we can further study the problem of optimal control strategy in different stages.

Vaccines for COVID-19 are currently available in many countries. Unfortunately, there are many different variations in COVID-19 that make the vaccine not fully effective against the virus. Therefore, it is also interesting to study the dynamics of how COVID-19 spreads differently in the presence of an imperfect vaccine. We leave the work for the future.

Acknowledgements The authors are grateful to the editor and the reviewers for their valuable comments and suggestions. This work was supported by Basic Competence Promotion Project for Young and Middle-aged Teachers in Guangxi, China (2019KY0269).

\section{Declarations}

Disclosure statement There are no conflicts of interest by the authors.

\section{References}

1. World Health Organization (WHO). Coronavirus. https://www.who.int/health-topics/coronavirus

2. World Health Organization (WHO). Situation report. https://www.who.int/zh/dg/speeches/detail/whodirector-general-s-remarks-at-the-media-briefing-on-2019-ncov-on-11-february-2020

3. World Health Organization (WHO). Situation report. https://www.who.int/zh/dg/speeches/detail/whodirector-general-s-opening-remarks-at-the-mission-briefing-on-COVID-19---12-march-2020

4. National Health Commission of the People's Republic of China. http://www.nhc.gov.cn/xcs/xxgzbd. shtml

5. Tang, B., Wang, X., et al.: Estimation of the transmission risk of 2019-nCov and its implication for public health interventions. J. Clin. Med. 9(462), 1-13 (2020)

6. Yan, Y., Chen, Y., Liu, K.J., et al.: Modeling and prediction for the trend of outbreak of NCP based on a time-delay dynamic system (in Chinese). Sci. Sin. Math. 50, 1-8 (2020)

7. Khan, M.A., Atangana, A.: Modeling the dynamics of novel coronavirus (2019-nCov) with fractional derivative. Alex. Eng. J. 59(4), 2379-2389 (2020)

8. Tang, B., Bragazzi, N.L., Li, Q., et al.: An updated estimation of the risk of transmission of the novel coronavirus(2019-nCov). Infect. Dis. Model. 5, 248-255 (2020)

9. Li, Q., Guan, X., Wu, P., et al.: Early transmission dynamics in Wuhan, China, of novel coronavirusinfected pneumonia. N. Engl. J. Med. 382(13), 1199-1207 (2020)

10. Cohen, J., Normile, D.: New SARS-like virus in China triggers alarm. Science 367(6475), 234-235 (2020)

11. Gilbert, M., Pullano, G., Pinotti, F., et al.: Preparedness and vulnerability of African countries against importations of COVID-19: a modelling study. Lancet 395(10227), 871-877 (2020)

12. Zhao, S., Lin, Q., et al.: Preliminary estimation of the basic reproduction number of novel coronavirus (2019-nCoV) in China, from 2019 to 2020: a data-driven analysis in the early phase of the outbreak. Int. J. Infect. Dis. 92, 214-217 (2020)

13. Khan, M.A., Ali, K., Bonyah, E., Okosun, K.O., Islam, S., Khan, A.: Mathematical modeling and stability analysis of Pine Wilt Disease with optimal control. Sci. Rep. 7(1), 3115 (2017)

14. Li, K., Zhu, G., Ma, Z., Chen, L.: Dynamic stability of an SIQS epidemic network and its optimal control. Commun. Nonlinear. Sci Numer. Simul. 66, 84-95 (2019)

15. Bonyah, E., Gmez-Aguilar, J.F., Adu, A.: Stability analysis and optimal control of a fractional human African trypanosomiasis model. Chaos Soliton Fract. 117, 150-160 (2018)

16. Huo, H., Yang, P., Xiang, H.: Stability and bifurcation for an SEIS epidemic model with the impact of media. Physica A. 490, 702-720 (2018)

17. Guo, Y., Li, T.: Optimal control and stability analysis of an online game addiction model with two stages. Math. Methods Appl. Sci. 43(7), 4391-4408 (2020) 
18. Bonyah, E., Khan, M.A., Okosun, K.O., Gomez-Aguilar, J.F.: Modelling the effects of heavy alcohol consumption on the transmission dynamics of gonorrhea with optimal control. Math. Biosci. 309, 1-11 (2019)

19. Driessche, P.V., Watmough, J.: Reproduction numbers and sub-threshold endemic equilibria for compartmental models of disease transmission. Math. Biosci. 180, 29-48 (2002)

20. Ełaiw, A.M., Raezah, A.A., Hattaf, K.: Stability of HIV-1 infection with saturated virus-target and infected-target incidences and CTL immune response. Int. J. Biomath. 10(05), 1750070 (2017)

21. Agusto, F.B., ELmojtaba, I.M.: Optimal control and cost-effective analysis of malaria/visceral leishmaniasis co-infection. PLoS One 12(2), e01721102 (2017)

22. LaSalle, J.P.: The stability of dynamical systems. In: Regional Conference Series in Applied Mathematics. SIAM, Philadelphia (1976)

23. Li, T., Guo, Y.: Optimal control of an online game addiction model with positive and negative media reports. J. Appl. Math. Comput. 66(1), 599-619 (2021)

24. Tang, S., Xiao, Y., Yuan, L., Cheke, R.A., Wu, J.: Campus quarantine (Fengxiao) for curbing emergent infectious diseases: lessons from mitigating A/H1N1 in Xi'an, China. J. Theor. Biol. 295, 47-58 (2012)

25. Heffernan, J.M., Smith, R.J., Wahl, L.M.: Perspectives on the basic reproductive ratio. J. R. Soc. Interface 2(4), 281-293 (2005)

26. National Health Commission of the People's Republic of China. http://www.nhc.gov.cn/guihuaxxs/ s10748/202006/ebfe31f24cc145b198dd730603ec4442.shtml

27. You, Z., Goh, S., Dong, J.: Predictive models for dynamic modulus using weighted least square nonlinear multiple regression model. Can. J. Civ. Eng. 39, 589-597 (2012)

28. Bogert, A., Su, A.: A weighted least squares method for inverse dynamic analysis. Comput. Methods Biomech. 359, 165-185 (2019)

29. Wang, L., Wang, J., Zhao, H., Shi, Y., Wang, K., Wu, P., Shi, L.: Modelling and assessing the effects of medical resources on transmission of novel coro-navirus (COVID-19) in Wuhan, China. Math. Biosci. Eng. 17(4), 2936-2949 (2020)

30. Mena-Lorca, J., Hetheote, H.W.: Dynamic models of infectious diseases as regulatiors of population sizes. J. Math. Biol. 30, 693-716 (1992)

31. Marino, S., Hogue, I.B., Ray, C.J., Kirschner, D.E.: A methodology for performing global uncertainty and sensitivity analysis in systems biology. J. Theor. Biol. 254(1), 178-196 (2008)

32. Khan, M.A., Shah, S.A.A., Ullah, S., Okosun, K.O., Farooq, M.: Optimal control analysis of the effect of treatment, isolation and vaccination on hepatitis B virus. J. Biol. Syst. 28(02), 351-376 (2020)

33. Das, D.K., Khajanchi, S., Kar, T.K.: The impact of the media awareness and optimal strategy on the prevalence of tuberculosis. Appl. Math. Comput. 366, 1244732 (2020)

34. Shi, R., Lu, T.: Dynamic analysis and optimal control of a fractional order model for hand-foot-mouth Disease. J. Appl. Math. Comput. 64, 565-590 (2020)

35. Sharomi, O., Gumel, A.B.: Curtailing smoking dynamics: a mathematical modeling approach. Appl. Math. Comput. 195, 475-499 (2008)

36. Bajeux, N., Ghosh, B.: Stability switching and hydra effect in a predator-prey metapopulation model. Biosystems 198, 104255 (2020)

37. Aziz-Alaoui, M.A., Okiye, M.D.: Boundedness and global stability for a predator-prey model with modified Leslie-Gower and Holling-Type II Schemes. Appl. Math. Lett. 16, 1069-1075 (2003)

38. Wu, Y., Huang, W.: Global stability of the predator-prey model with a sigmoid functional response. Discrete Contin. Dyn. B 25(3), 1159-1167 (2020)

39. Aba Oud, M.A., Ali, A., Alrabaiah, H., Ullah, S., Khan, M.A., Islam, S.: A fractional order mathematical model for COVID-19 dynamics with quarantine, isolation, and environmental viral load. Adv. Differ. Equ. 2021(1), 106 (2021)

40. Gupta, M., Mohanta, S.S., Rao, A., Parameswaran, G.G., Agarwal, M., Arora, M., et al.: Transmission dynamics of the COVID-19 epidemic in India and modeling optimal lockdown exit strategies. Int. J. Infect. Dis. 103, 579-589 (2021)

41. Hezam, I.M., Foul, A., Alrasheedi, A.: A dynamic optimal control model for COVID-19 and cholera co-infection in Yemen. Adv. Differ. Equ. 2021(1), 108 (2021)

42. Wickramaarachchi, W.P.T.M., Perera, S.S.N.: An SIER model to estimate optimal transmission rate and initial parameters of COVD-19 dynamic in Sri Lanka. Alex. Eng. J. 60(1), 1557-1563 (2021)

43. Gmez-Aguilar, J.F., Alderremy, A.A., Aly, S., Saad, K.M.: A fuzzy fractional model of coronavirus (COVID-19) and its study with Legendre spectral method. Results Phys. 21, 103773 (2021) 
44. Rahman, M.U., Arfan, M., Shah, K., Gmez-Aguilar, J.F.: Investigating a nonlinear dynamical model of COVID-19 disease under fuzzy caputo, random and ABC fractional order derivative. Chaos Soliton Fract. 140, 110232 (2020)

45. Panwar, V.S., Uduman, P.S.S., Gmez-Aguilar, J.F.: Mathematical modeling of coronavirus disease COVID-19 dynamics using $\mathrm{CF}$ and $\mathrm{ABC}$ non-singular fractional derivatives. Chaos Soliton Fract. 145, 110757 (2021)

46. Chen, S.B., Rajaee, F., Yousefpour, A., Alcaraz, R., Chu, Y.M., Gmez-Aguilar, J.F., et al.: Antiretroviral therapy of HIV infection using a novel optimal type-2 fuzzy control strategy. Alex. Eng. J. 60(1), 15451555 (2021)

47. Hussain, T., Aslam, A., Ozair, M., Tasneem, F., Gmez-Aguilar, J.F.: Dynamical aspects of pine wilt disease and control measures. Chaos Soliton Fract. 145, 110764 (2021)

Publisher's Note Springer Nature remains neutral with regard to jurisdictional claims in published maps and institutional affiliations. 